\title{
Erratum to: Being "in Control" May Make You Lose Control: The Role of Self-Regulation in Unethical Leadership Behavior
}

\author{
Anne Joosten - Marius van Dijke - Alain Van Hiel • \\ David De Cremer
}

Published online: 25 May 2013

(C) Springer Science+Business Media Dordrecht 2013

\section{Erratum to: J Bus Ethics}

DOI 10.1007/s10551-013-1686-2

Table 4 in the above mentioned article should read:

Table 4 Results of hierarchical regression analysis for leader deviance (colleague indicated) in Study 2

\begin{tabular}{lrrrr}
\hline Variables & \multicolumn{1}{c}{$B$} & $S E B$ & $\beta$ & $\Delta R^{2}$ \\
\hline Step 3 & & & & $.10^{* * * *}$ \\
Age & -0.03 & 0.01 & $-0.30^{* * *}$ & \\
Gender & -0.07 & 0.16 & -0.04 & \\
Organization tenure & 0.01 & 0.01 & 0.13 & \\
Function tenure & -0.00 & 0.02 & -0.02 & \\
Regulatory depletion (RD) & 0.19 & 0.08 & $0.22^{*}$ & \\
Moral identity (MI) & -0.13 & 0.09 & -0.13 & \\
RD $\times$ MI & -0.32 & 0.09 & $-0.33^{* * *}$ &
\end{tabular}

Final model: $F(7,92)=4.36, p<001 . B=$ unstandardized regression coefficient; $\beta=$ standardized regression coefficient

$* p \leq .05 ; * * p \leq .01 ; * * * p \leq .001$

The online version of the original article can be found under doi:10.1007/s10551-013-1686-2.

A. Joosten $(\bowtie) \cdot$ A. Van Hiel

Department of Developmental, Personality and Social

Psychology, Ghent University, Henri Dunantlaan 2, 9000 Ghent,

Belgium

e-mail: anne.joosten@ugent.be

M. van Dijke

Rotterdam School of Management, Erasmus University,

Burgemeester Oudlaan 50, 3062 PA Rotterdam, The Netherlands

D. De Cremer

CEIBS, 699 Hongfeng Road, Shanghai 201206, Pudong, China
More specifically, the asterisks following the Beta values are given correctly in this table. 